\title{
Familiarity does not Inhibit Image-Specific Encoding of Faces
}

\author{
James D. Dunn \\ UNSW Sydney \\ Kay L. Ritchie \\ University of Lincoln \\ Richard I. Kemp and David White \\ UNSW Sydney
}

\section{Please cite this pre-print as:}

Dunn, J. D., Ritchie, K. L., Kemp, R. I. \& White. D. (in press). Familiarity does not inhibit image-specific encoding of faces in short-term memory. Journal of Experimental Psychology: Human Perception and Performance.

(C) 2018, American Psychological Association. This paper is not the copy of record and may not exactly replicate the final, authoritative version of the article. Please do not copy or cite without authors' permission. The final article will be available, upon publication, via its DOI: $10.1037 / \mathrm{xhp} 0000625$ 


\section{Author Note}

James D. Dunn, School of Psychology, UNSW Sydney, j.d.dunn@,unsw.edu.au; Kay L. Ritchie, School of Psychology, University of Lincoln, kritchie@lincoln.ac.uk; Richard I. Kemp, School of Psychology, UNSW Sydney, richard.kemp@unsw.edu.au; David White, School of Psychology, UNSW Sydney, david.white@unsw.edu.au.

This research was supported by an Australian Government Research Training Program Scholarship awarded to Dunn and by Australian Research Council Linkage Project grants to White and Kemp (LP130100702, LP160101523) and by funding from the Australian Passport Office. The authors thank Rebekah Au for her assistance in collecting data for Experiment 5. Experiment data, materials and program scripts can be downloaded from https://github.com/UNSWfacelab/dunnetal_JEP_HPP_materials.

Correspondence concerning this article should be addressed to David White, School of Psychology, UNSW Sydney, 2052, Australia. Email: david.white@unsw.edu.au 


\begin{abstract}
(178 words)
When matching and recognising familiar faces, performance is unaffected by changes to image-specific details such as lighting, head angle, and expression. In contrast, these changes have a substantial impact on performance when faces are unfamiliar. What process can account for this difference? Recent evidence shows a memory disadvantage for remembering specific images of familiar people compared to unfamiliar people, suggesting that image invariance in familiar face processing may be supported by loss of image-specific details in memory. Here, we examine whether this cost results from loss of image specific details during encoding of familiar faces. Participants completed four tasks that required participants to retain image-specific information in working memory: duplicate detection (Experiment 1), change detection (Experiment 2), short-term recognition memory (Experiment $3 \& 5$ ), and visual search (Experiment 4). Across all experiments (combined $n=270$ ), our results consistently show equivalent memory performance for specific images of familiar and unfamiliar faces. We conclude that familiarity does not influence encoding of pictorial details, suggesting that loss of image-specificity reported in previous work is a result of longer-term storage mechanisms.
\end{abstract}

Keywords: face recognition; face perception; representation; predictive coding; visual working memory. 
Public Significance Statement (98 words)

We recognise familiar faces despite substantial variation in appearance from one encounter to the next. How perceptual systems achieve accurate recognition despite this variation remains unclear. One proposal is that the perceptual system represents abstract visual properties of familiar faces by discarding 'image-specific' details such as a particular lighting quality or head pose. Here, we test this hypothesis by comparing participants' short-term memory for specific images of familiar and unfamiliar faces. We find that performance is unaffected by familiarity and conclude that image-specific details are encoded in memory to an equivalent extent for both familiar and unfamiliar faces. 
Familiarity does not Inhibit Image-Specific Encoding of Faces

People are able to match images of familiar faces despite large changes in appearance due to age, lighting, viewpoint, and degradation in image quality (e.g. Bruce, Henderson, Newman, \& Burton, 2001; Burton, Wilson, Cowan, \& Bruce, 1999; Johnston \& Edmonds, 2009; Megreya \& Burton, 2007). In contrast, even under optimal conditions where changes in appearance are minimised, unfamiliar face matching is much more difficult (e.g. Bruce et al., 1999; Burton, White, \& McNeill, 2010; Megreya \& Burton, 2006, 2008; Ritchie et al., 2015). Leading accounts of this difference propose that recognition of familiar faces is facilitated by enhanced view-independent structural codes (Bruce \& Young, 1986), whereas unfamiliar faces identification relies on view-specific pictorial codes which encode properties that are specific to the image encountered (Longmore, Liu, \& Young, 2008; Megreya \& Burton, 2006).

These accounts provide a framework for understanding people's ability to recognise familiar faces despite substantial variability in appearance. In a striking demonstration of this ability, Jenkins, White, Van Montfort, and Burton (2011) asked participants to sort forty images into piles by identity. The images were of two people, but when participants were unfamiliar with these people, they sorted the images into an average of seven different piles; demonstrating a strong tendency to misinterpret changes in image as changes in identity. Participants familiar with the face almost always arrived at the correct solution, sorting the images into two piles. This finding suggests that when a face becomes familiar, our perception becomes less influenced by image-specific information (see also Balas \& Pearson, 2017; Baker, Laurence \& Mondloch, 2017; Bruce, 1982; Bruce \& Young, 1986; Burton, Jenkins, Hancock, \& White, 2005; Burton, Kramer, Ritchie, \& Jenkins, 2016; Calder \& Young, 2005; Johnston \& Edmonds, 2009). 
Familiarity produces representations that are tolerant of variation in photos of the same face, but the processes that underpin this are not fully understood. In one account, Bruce (1994) proposed that our visual system accommodates variation in the appearance of faces by forming abstract representations that aggregate information across different encounters with a face. By accumulating variability, she proposed, the visual system derives a representation containing features that do not vary across images of the same face and are therefore diagnostic of identity. Burton et al. (2005) extended this idea, modelling the abstractive representation as a statistical average of multiple images of the same face. In subsequent empirical work, face averages have been shown to produce improved unfamiliar face recognition relative to single images (Burton et al., 2005; White, Burton, Jenkins, \& Kemp, 2014).

According to these theories, memory representations supporting face recognition rely on abstraction from properties that are specific to a given encounter — or image - of a face. One prediction that arises from these accounts is that familiarity should result in a loss of image-level detail when photographs of familiar faces are stored in memory. To test this, Armann, Jenkins, and Burton (2016) examined recognition for specific images of familiar and unfamiliar faces. In their strongest test of this hypothesis, participants studied images of faces and were instructed that they would be required to remember the specific image that they had studied. Crucially, this task relied on their memory for the unique superficial features that distinguished each image rather than the identity of the face in the image. Participants were less accurate at remembering images they had seen when faces were familiar compared to those that were unfamiliar, pointing to less pictorial detail in long-term memory representations of familiar faces. The authors interpret this as a qualitative change in the processing of image information for familiar faces, rather than a simple accumulation of more information over time. 
Despite this intriguing finding, the nature of differences between pictorial level processing of familiar and unfamiliar faces remains unclear. Because Armann et al. (2016) tested only long-term recognition memory, it is not clear whether familiarity produces differences in the long-term retention of image-specific details, or whether it causes changes in the initial encoding of faces.

The latter explanation is plausible in light of evidence showing that familiarity can influence encoding at the earliest stages of visual processing (for review see: Ramon \& Gobbini, 2018). For example, familiarity is known to lead to speeded face detection (Visconti di Oleggio Castello \& Gobbini, 2015), facilitated processing of head angle and eye-gaze direction (Visconti di Oleggio Castello, Guntupalli, Yang \& Gobbini, 2014) and faster, more automatic encoding of face gender and identity information (Balas, Cox \& Conwell \& 2007; Rossion, 2002; Yan, Young \& Andrews, 2017). Reduced encoding of image-specific properties may also explain people's reduced ability to discriminate between images of their own face (White, Sutherland \& Burton, 2017). Together with evidence that early electrophysiological markers of face encoding are sensitive to familiarity (Caharel, Ramon \& Rossion, 2014; Colins, Robinson \& Behrmann, 2018; c.f. Johnston et al. 2016), these results suggest that familiar faces may be processed differently to unfamiliar faces during early stages.

Here, we test whether deficits found in image memory for familiar faces are caused by differences in initial encoding of pictorial information. We compared matching performance for identical images of familiar and unfamiliar faces using four tasks that relied on encoding image-specific details in working memory: Duplicate detection (Experiment 1), change detection (Experiment 2), short-term recognition memory (Experiments 3 \& 5), and visual search (Experiment 4). These paradigms were selected because they all target early levels of perceptual representation, and because they show an advantage for familiar faces on 
tasks that require matching the identity of a target (e.g. Duplicate detection: Chapman \& Susilo, 2018; Change detection/Short-term memory: Jackson \& Raymond, 2008; Visual search: Tong \& Nakayama, 1999). In our study however, as with the long-term image memory task used by Armann and colleagues (2016), we modify these tasks by requiring participants to match the precise image rather than the identity. By using a variety of paradigms that rely on encoding of image-level information, we aimed to provide converging evidence as to whether these early representations are affected by face familiarity.

\section{Experiment 1}

In Experiment 1, participants' task was to view arrays of images and decide whether the array contained a duplicate image. This task required participants to accurately encode image-level information. To ensure that differences between familiar and unfamiliar conditions were due to familiarity, rather than the particular image sets used in the study, we recruited UK and Australian participants. This enabled us to counterbalance the celebrities that were familiar across these participant groups (see Design and Procedure). Based on the results reported by Armann and colleagues (2016), we predicted poorer duplicate detection performance when presented with an array of familiar face images than when presented with an unfamiliar face.

\section{Method}

Sample size estimation. We performed a statistical power analysis using GPower 3.1 software (Erdfelder, Faul \& Buchner, 1996) based on accuracy data from Armann et al. (2016). In this study, an effect size of $\eta p^{2}=0.15$ (Experiment 1) and 0.3 (Experiment 2) was reported for the advantage of unfamiliar faces over familiar faces. Taking the smallest effect size estimate, which corresponds to Cohen's $f=0.42$, a projected sample size of 48 participants was needed to detect an effect of this size with the simplest within-subjects 
comparison $(\alpha=.05$, power $=0.8)$. Consequently, in all studies reported here we recruited a minimum number of 48 participants once accounting for exclusions based on other eligibility criteria (see Stimuli and Procedure sections).

Participants. Forty-three undergraduate students from the University of New South Wales (Australian participants: 24 female, mean age 19.7, $S D=3.5$ ) and 28 undergraduate students from the University of York (UK participants: 22 female, mean age 20.0, $S D=3.0$ ) took part in the experiment in exchange for course credit. Of the 43 University of New South Wales students, 15 did not meet the criteria for inclusion (see Design and procedure). This left a final sample of 28 undergraduate students from the University of New South Wales (Australian participants: 16 female, mean age 19.0, $S D=1.1$ ) along with the 28 undergraduate students from the University of York (UK participants: 22 female, mean age $20.0, S D=3.0)$

Stimuli. Stimuli consisted of images of 40 UK celebrities and 40 Australian celebrities. These individuals were 'local' celebrities — for example TV presenters, sports personalities, and politicians - chosen to be known locally in either Australia or the UK, but not in both countries. For each identity, we downloaded 25 unique images from Google Image Search. Images were cropped around the head to remove the background using a rectangular marquee with aspect ratio 3:4 and then rescaled to 150x200 pixels. All images showed the face in full portrait view, but otherwise were sampled to vary naturally in factors such as lighting, expression, pose, and head angle (see Jenkins et al., 2011).

Design and procedure. The experiment was implemented in Psychtoolbox for MATLAB (Brainard, 1997). First, we determined participants' pre-existing familiarity with each of the Australian and UK celebrities by showing the names of all celebrities and asking 
participants to respond 'familiar' when they recognised the name of the person and could imagine what they looked like. These responses were then used to determine the subset of celebrities that were included in familiar and unfamiliar conditions of the duplicate detection task. Performing this questionnaire before beginning the experiment ensured that the familiar and unfamiliar stimulus sets could be tailored to individual participants. UK celebrities labelled as familiar and Australian faces labelled as unfamiliar were not shown to Australian participants and vice-versa for UK participants. Because each participant viewed 30 familiar and 30 unfamiliar arrays, for participants familiar with less than $30(75 \%)$ of celebrities it was necessary to repeat some familiar target faces multiple times during the experiment. For participants familiar with more than 30 , a random subset of 30 familiar faces were selected.

Only participants familiar with at least 10 celebrities and unfamiliar with at least 10 celebrities were included in the study, meaning that 15 participants who completed the study were not included in the final analysis. The remaining participants were on average familiar with $73 \%$ of the 'familiar' celebrities and $2 \%$ of 'unfamiliar' celebrities. Importantly, we found that this variability in the number of celebrities each participant recognised did not affect the conclusions of any experiment in this paper (see Supplementary Materials).

On each trial, participants were shown an array of images in the centre of the screen presented in a 2 by 2 ( 4 images), 3 by 3 (9 images) or 4 by 4 (16 images) grid. Participants were instructed to decide whether there were duplicate images present in each array (i.e. any two images that were exact copies of each other, see Figure 1). They were also informed that all images shown on the same screen were of the same person. On duplicate present trials, all images within an array were unique except for a single duplicate image pair. On duplicate absent trials, all images within an array were unique. A duplicate image pair was present on $50 \%$ of trials and the images within the array were always presented in a random 
configuration. Participants had to respond by key press to indicate whether there was a duplicate present and were instructed to respond as quickly and as accurately as possible.

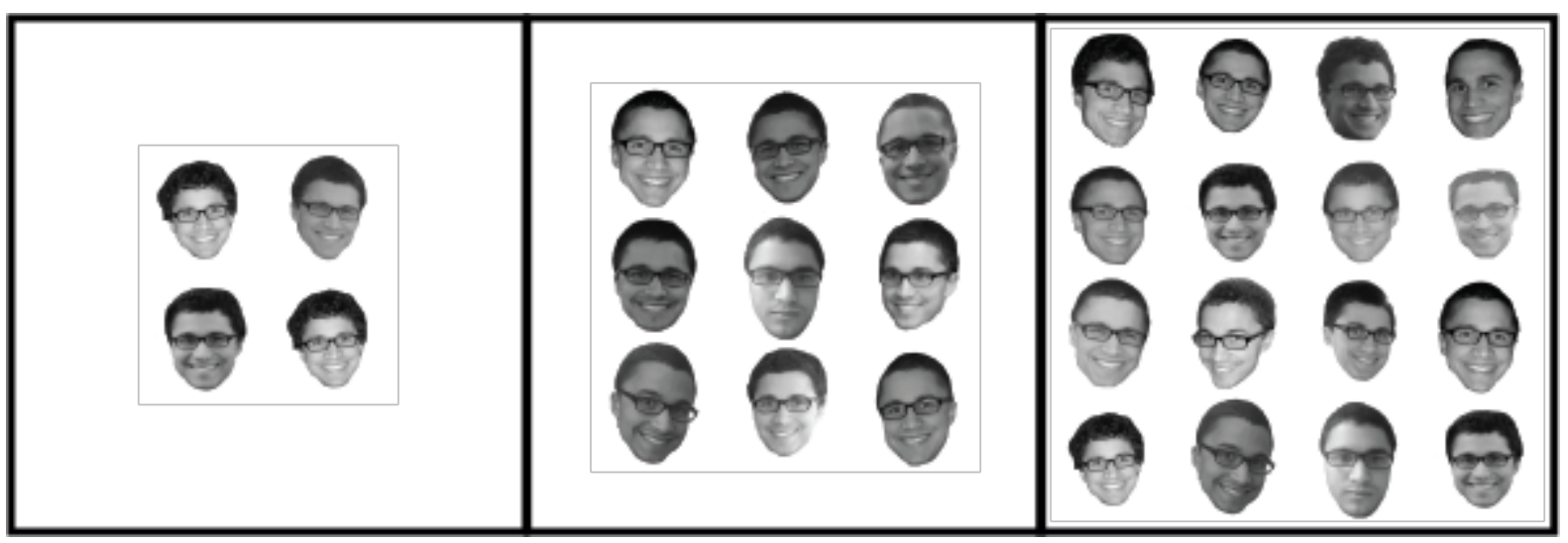

Figure 1. Example arrays of each size used in Experiment 1. Participants had to decide whether a duplicate image was present in the array. Images are representative of the stimuli used in the matching task, but for copyright restrictions, we are not able to provide the actual stimuli used in our studies. Correct locations of duplicates are at grid locations 1,1 and 2,2 (left); no duplicate (middle); 2,2 and 4,4 (right).

Participants completed 2 practice trials before attempting the main task, which consisted of 60 trials split into 6 blocks of equal length. Block order was randomised for each participant. Each block had trials of the same conditions, so that in each block participants saw either an unfamiliar face or familiar face presented in a 4, 9 or 16 image array.

\section{Results}

Performance on the duplicate detection task was measured using signal detection theory parameters sensitivity $\left(d^{\prime}\right)$ and criterion $(C)$ and is shown in Figure 2. Because we were interested in whether perceptual encoding of images is impaired for familiar faces, the measure of primary interest was sensitivity. However, we also analysed criterion for evidence of response bias ${ }^{1}$. For the analysis of sensitivity, hits were defined as duplicate-present trials 
in which the participant responded correctly, and false alarms were trials in which there was not a duplicate pair, but participants incorrectly responded that a duplicate was present. For criterion, positive values indicate a greater tendency to respond "no duplicate".

Both signal detection measures were analysed in a $2 \times 3$ repeated measures ANOVA with Familiarity (familiar, unfamiliar) and Array Size (4 images, 9 images, 16 images) as the within participant factors. We also examined the strength of evidence for the critical main effect of Familiarity using JASP (version 0.9.0.1) to estimate a Bayes factors based on Bayesian Information Criteria (see Wagenmakers, 2007).
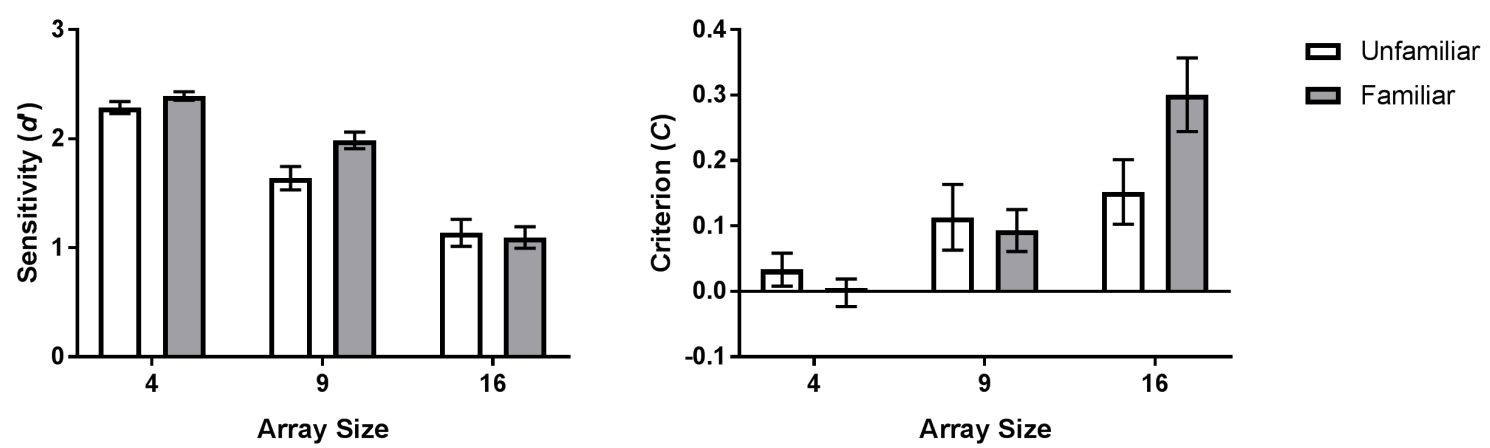

Figure 2. Duplicate detection performance. Sensitivity (d') and response criterion $(C)$ for Experiment 1. Error bars denote \pm 1 standard error.

Sensitivity. For sensitivity scores, the main effect of Familiarity was non-significant, $F(1,55)=3.572, p=.064, \eta_{\mathrm{p}}{ }^{2}=.061$, with a trend for familiar arrays to produce superior performance (i.e. a mean difference in the opposite direction to predicted). The main effect of Array Size was significant, $F(2,110)=120.21, p<.001, \eta_{\mathrm{p}}{ }^{2}=.686$, with means showing a decrease in accuracy with increasing array size. Post-hoc tests showed that performance significantly declined from Array Size, 4 v 9, $t(55)=-7.116, p<.001$, Cohen's $d=-1.079$, and between $9 \mathrm{v} 16, t(55)=-7.786, p<.001$, Cohen's $d=-1.041$. The two-way interaction between Array size and Familiarity was not significant, $F(2,110)=2.955, p=.061, \eta_{\mathrm{p}}{ }^{2}=$ .051 . 
The estimated Bayes factor suggested these data were 2.56:1 in favour of the null hypothesis, implying that these results are 2.56 times more likely to occur in a model without familiarity as a factor than a model that includes it. This provided preliminary support in favour of the null hypothesis, suggesting that familiarity does not affect sensitivity on this task.

Criterion. For criterion scores, the main effect of Familiarity was non-significant, $F(1,55)=1.167, p=.285, \eta_{\mathrm{p}}{ }^{2}=.021$. The main effect of Array Size was significant, with mean differences showing a greater bias to respond "No Duplicate" with increasing Array Size, $F(2,110)=12.341, p<.001, \eta_{\mathrm{p}}^{2}=.183$. The interaction between factors was significant, $F(2,110)=3.843, p=.030, \eta_{\mathrm{p}}^{2}=.065$. Planned comparisons show this interaction was driven by a significant difference in criterion between unfamiliar and familiar faces for array size 16, with participants less likely to respond present in familiar compared to unfamiliar face arrays, $t(55)=2.353, p=.022$, Cohen's $d=0.635$.

\section{Discussion}

In Experiment 1, we tested whether familiarity affected performance on a perceptual matching task that required participants to detect duplicate images in an array. For the critical measure of perceptual sensitivity, we found weak evidence to support the null hypothesis. Given that performance for duplicate detection of familiar face images was numerically superior to unfamiliar face images, this provides preliminary evidence that the cost of familiarity found for long-term image memory tasks is not caused by differences in encoding of familiar and unfamiliar faces. Importantly however, in the duplicate detection task all images appeared on the screen at once and participants were able to return their gaze to all images as often as they liked in order to maximise accuracy. Thus, it is possible that we found no effect of familiarity because the duplicate detection task had minimal memory 
demands. Therefore, in the next experiment we examined whether image memory would be sensitive to familiarity in a task that required participants to remember specific images across short retention intervals.

\section{Experiment 2}

In Experiment 2, we used a change detection task to determine whether familiarity affected encoding of superficial image details in working memory. Using this paradigm, Jackson and Raymond (2008) showed a short-term memory advantage for familiar over unfamiliar faces when the task required participants to detect a change in identity of one of the faces. Here, participants studied image arrays of familiar and unfamiliar faces, but the identity of faces did not change between study and test, instead participants had to decide whether there had been a change in the precise image between study and test.

\section{Method}

Participants. Because the effects of familiarity did not differ between Australian and UK participants in Experiment 1, we recruited only Australian participants in the experiment. One hundred undergraduate students from the University of New South Wales (68 female, mean age 19.0, $S D=1.9$ ) took part in the experiment in exchange for course credit. Of these, 44 did not meet the criteria for inclusion. This left a final sample of 56 undergraduate students from the University of New South Wales (Australia) (32 female, mean age 18.8, SD $=1.5)$.

Stimuli, design and procedure. We used the same stimuli and pre-screening familiarity questionnaire as in Experiment 1. Again, only participants familiar with at least 10 celebrities and unfamiliar with at least 10 celebrities were included in the study. Due to the local Australian celebrities being unfamiliar to a substantial proportion of undergraduate 
students, 44 participants who completed the study were not included in the final analysis. The remaining participants were on average familiar with $57 \%$ of the 'familiar' celebrities and $4 \%$ of 'unfamiliar' celebrities.

The trial structure is illustrated in Figure 3. On each trial, participants were instructed to remember the array of images, with array size varying so that there were 4,6 or 8 images on screen simultaneously. These images were presented onscreen for 4 seconds, followed by a blank screen for 1 second, after which the test image appeared and participants had to respond whether the test image matched the image that had previously appeared at this location. The test image was randomly selected from the array images and all images within an array were unique images of the same identity. Participants were instructed to respond as quickly and as accurately as possible.

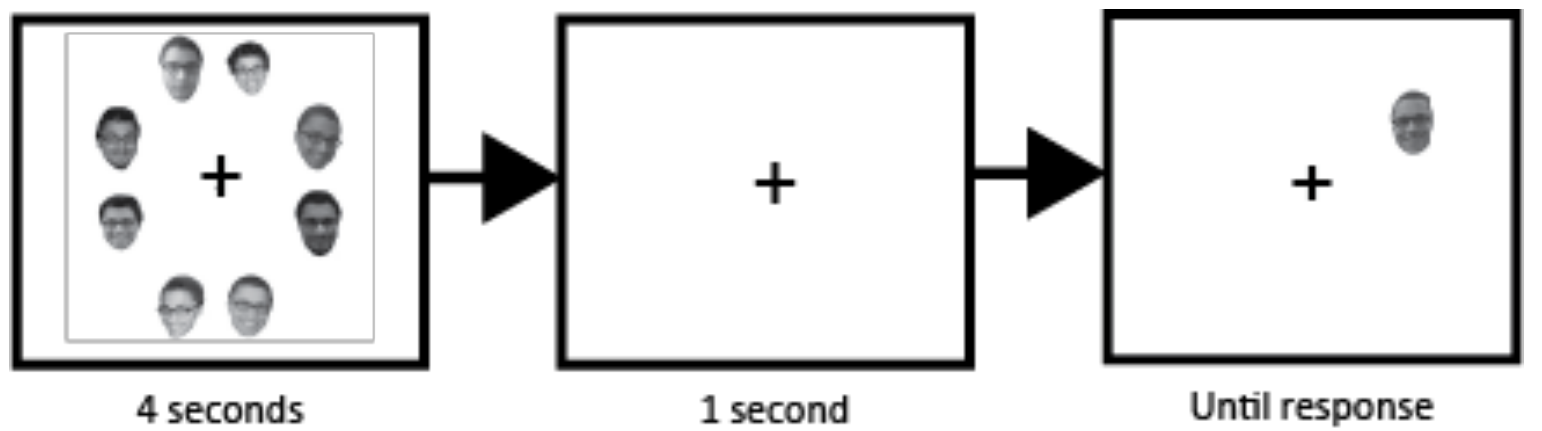

Figure 3. The change detection paradigm employed used in Experiment 2.

Participants studied an array of images for 4 seconds and then had to indicate whether a probe image matched the image, which had been at the same location (in this trial the correct response would be 'Different').

As with Experiment 1, participants completed 2 practice trials before attempting the main task, which consisted of 60 trials split into 6 blocks of equal length. Block order was randomised for each participant. Both the array size and the familiarity of the identity in the 
array remained the same within a block. On $50 \%$ of trials, the test image changed between presentations, with the remaining trials having the same image in the target location.

\section{Results}

Sensitivity and criterion scores are shown in Figure 4. These data were analysed separately using a 2x3 repeated measures ANOVA with Familiarity (familiar, unfamiliar) and Array Size (4 images, 6 images, 8 images) as the within participant factors.
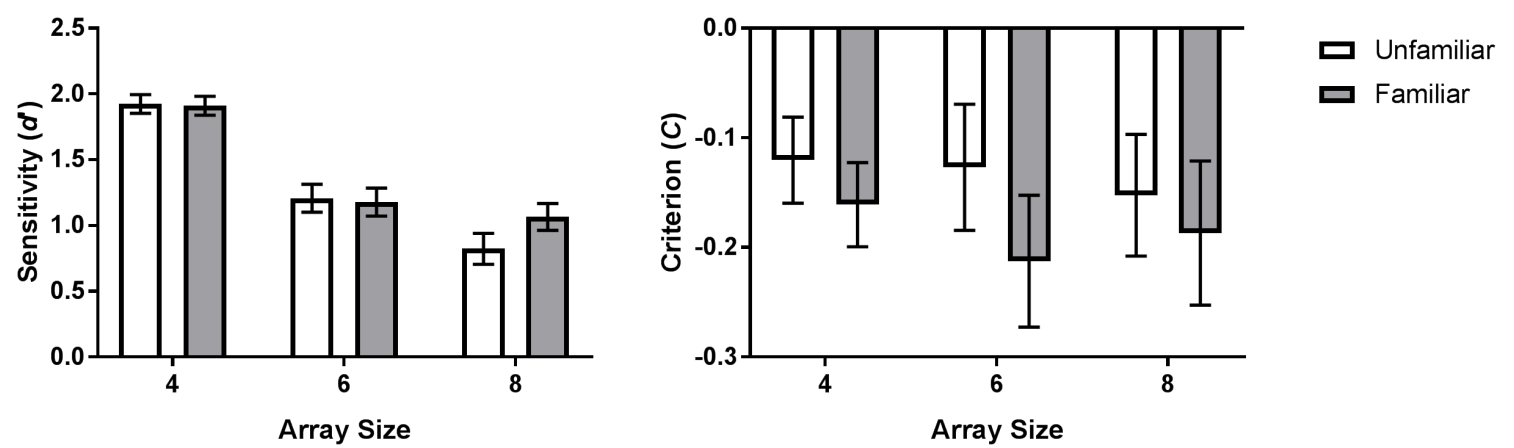

Figure 4. Change detection performance. Sensitivity (d') and response criterion (C) for Experiment 2. Error bars denote \pm 1 standard error.

Sensitivity. For sensitivity the main effect of Familiarity was not significant, $F(1,55)$ $=0.786, p=.379, \eta_{\mathrm{p}}^{2}=.014$. There was a significant main effect of Array Size, $F(2,110)=$ $60.124, p<.001, \eta_{\mathrm{p}}^{2}=.522$, with performance significantly declining at each level of Array Size, 4 v 6, $t(62)=-7.041, p<.001$, Cohen's $d=-0.901 ; 6$ v $8, t(62)=-3.478, p=.001$, Cohen's $d=-0.438$. These was a non-significant interaction between these factors, $F(2,110)$ $=1.476, p=.233, \eta_{\mathrm{p}}{ }^{2}=.026$. We again examined the effect of familiarity on sensitivity by estimating a Bayes factor, which suggested the data were 7.14 times more likely to occur in a model without familiarity as factor than one with it. This finding provides support for the null hypothesis that familiarity does not affect sensitivity on this task. 
Criterion. Criterion data was analysed in the same way, indicating non-significant main effects of Familiarity, $F(1,55)=1.596, p=.212, \eta_{\mathrm{p}}^{2}=.028$, Array Size, $F(2,110)=$ $0.240, p=.787, \eta_{\mathrm{p}}{ }^{2}=.004$, and a non-significant interaction between factors, $F(2,110)=$ $0.153, p=.856, \eta_{\mathrm{p}}^{2}=.003$.

\section{Discussion}

The results of Experiment 2 show that participants' memory for specific images was unaffected by the familiarity of the face being viewed, using a task in which familiarity had previously been shown to facilitate identity processing (Jackson \& Raymond, 2008). This result extends the findings of Experiment 1, by suggesting that familiarity does not affect the encoding of pictorial information. However, as with Experiment 1, the change detection paradigm involves only immediate memory. Differences between image memory for familiar and unfamiliar faces may only emerge with longer retention intervals, and so we tested this in the next experiment.

\section{Experiment 3}

In Experiment 3, we test whether there are differences in image-level representation of familiar and unfamiliar faces using a short-term recognition memory paradigm. As with our previous experiments, we tested participant's ability to recognise whether a particular image was one they had studied on a previous screen. To ensure that accurate performance required retention of image-level information in visual working memory, we increased the delay between the array and test images and included a backwards visual mask to prevent reliance on sensory representations. 


\section{Method}

Participants. Forty-two undergraduate students from the University of New South Wales (Australian participants: 21 female, mean age 19.4, $S D=1.9$ ) and 27 undergraduate students from the University of Lincoln (UK participants: 19 female, mean age 21.1, SD= 3.4) took part in the experiment in exchange for course credit ${ }^{2}$. Of the 42 University of New South Wales students, 18 did not meet the criteria for inclusion. This left a final sample of 24 undergraduate students from the University of New South Wales (12 female, mean age 19.3, $S D=1.5)$ and 27 undergraduate students from the University of Lincoln (19 female, mean age 21.1, $S D=3.4)$. As in Experiment 1, this study tested participants from Australia and the UK to counterbalance familiarity whilst using the same stimuli set.

Stimuli, design and procedure. We used the same stimuli and pre-screening familiarity questionnaire as in Experiment 1 and 2. Again, only participants familiar with at least 10 celebrities and unfamiliar with at least 10 celebrities were included in the study, meaning that 18 participants who completed the study were not included in the final analysis. The remaining participants were on average familiar with $67 \%$ of the 'familiar' celebrities and $4 \%$ of 'unfamiliar' celebrities.

The trial structure is illustrated in Figure 5. In the study phase of each trial, participants were presented with arrays of either 1,2, 4, or 8 images, shown for 1, 2, 4, and 8 seconds respectively. All images in an array were unique images of the same identity. After the study period had elapsed, the array was replaced by a visual mask, which remained on screen for 1 second. Following this, participants were shown a blank screen for a further 3 seconds. Finally, a test image appeared, and participants were asked to indicate whether this image had appeared in the array. 


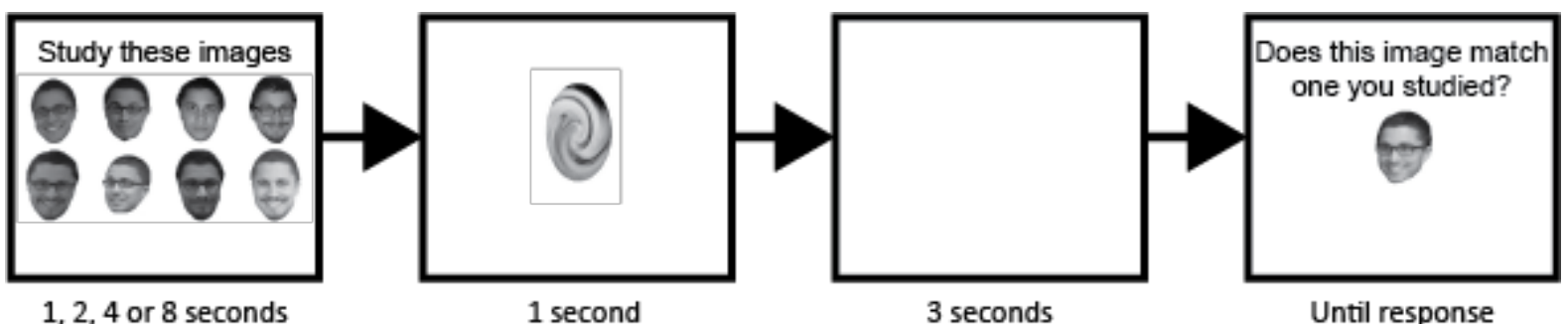

Figure 5. Short-term recognition memory paradigm used in Experiment 3.

Participants were shown an array of images to remember and had to indicate whether the test image was in the study array (in this trial the correct response would be 'Different').

Participants completed 2 practice trials before attempting the main task, which consisted of 80 trials split into 8 blocks of equal length. Block order was randomised for each participant. Both the array size and the familiarity of the identity in the array remained the same within a block. Half of trials in each block were 'match' trials, where the probe image was identical to one of the array images, and the other half were 'non-match' where the probe image was a novel image of the same face.

\section{Results}

Both signal detection measures were analysed in a 2x4 repeated measures ANOVA with Familiarity (familiar, unfamiliar) and Array Size (1 image, 2 images, 4 images, 8 images) as the within participant factors (Figure 6).

Sensitivity. For sensitivity, the main effect of Familiarity was not significant, $F(1,50)$ $=0.508, p=.479, \eta_{\mathrm{p}}^{2}=.010$. There was a significant main effect of Array Size, $F(3,150)=$ $88.666, p<.001, \eta_{\mathrm{p}}{ }^{2}=.639$, with performance significantly declining at each level of Array Size, 1 v 2: $t(50)=-7.777, p<.001$, Cohen's $d=-1.141 ; 2$ v 4: $t(50)=-4.268, p<.001$, Cohen's $d=-0.598,4 \mathrm{v} 8: t(50)=-5.100, p<.001$, Cohen's $d=-0.716$. The interaction between these factors was not significant, $F(3,150)=0.991, p=.395, \eta_{\mathrm{p}}^{2}=.019$. Looking specifically at the impact of familiarity on sensitivity, the estimated Bayes factor for this data 
suggested this pattern of results was 7.58 times more likely to occur in a model without familiarity as a factor than a model including familiarity, providing support for the null hypothesis that familiarity does not affect sensitivity on this task.
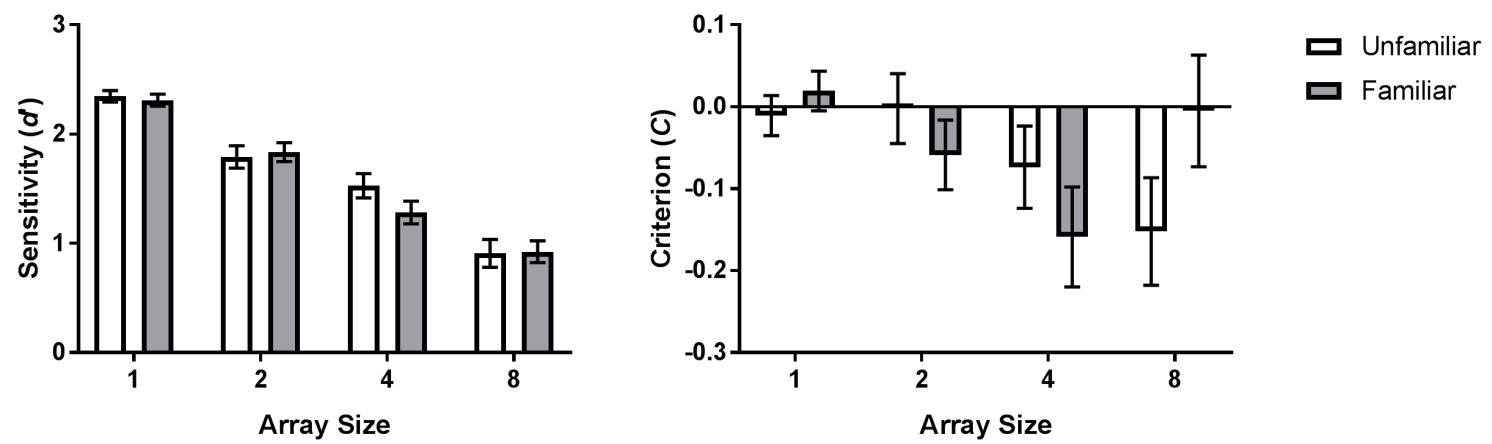

Figure 6. Performance on short-term image memory paradigm. Sensitivity (d') and response criterion (C) for Experiment 3. Error bars denote \pm 1 standard error.

Criterion. For criterion, we found non-significant main effects of familiarity, $F(1,50)$ $=0.057, p=.813, \eta_{\mathrm{p}}{ }^{2}=.001$, and Array Size, $F(3,150)=2.578, p=.063, \eta_{\mathrm{p}}{ }^{2}=.049$, and a non-significant interaction between factors, $F(3,150)=2.812, p=.054, \eta_{\mathrm{p}}{ }^{2}=.053$.

\section{Discussion}

The results of Experiment 3 again provided moderate support for the null hypothesis that participants' memory for specific images was unaffected by familiarity, despite being retained for a longer ( 4 second) interval. In combination with the previous experiments, this suggests that familiarity does not inhibit the encoding of image-specific details in working memory. In the next experiment, we test whether familiarity affects the ability to search for specific images of a face.

\section{Experiment 4}

Visual search paradigms have been shown to provide a sensitive tool for examining the specificity of working memory representations supporting performance (Bravo \& Farid, 
2009, 2012; Hout \& Goldinger, 2015; Stroud, Menneer, Cave \& Donnelley, 2012). Similar to the paradigms used in our previous experiments, search paradigms have also been used to demonstrate the benefits of familiarity when searching for images of a particular face identity (Dunn, Kemp, \& White, 2018; Ito \& Sakurai, 2014; Tong \& Nakayama, 1999). Here we ask whether familiarity affects how well participants search for a particular face image, where distractors are other images of the same person.

We also aimed to make the task challenging by requiring participants to search for multiple instances of a single target image. This task required participants to complete an exhaustive search for multiple targets, meaning that they had to find all of the instances of the targets in the search display (for description of exhaustive search, see Biggs, 2017). In addition, we included a condition where participants searched for two target images. Searching for two faces is known to produce substantial impairment due to capacity limits for unfamiliar faces (Mestry, Menneer, Cave, Godwin, \& Donnelly, 2017). Importantly, the magnitude of the dual-target cost is believed to be moderated by familiarity, where multiple highly familiar objects may be searched for with smaller costs to performance (e.g. Shiffrin \& Schneider, 1977). If long-term memory representations interact with image-specific representations, then this benefit of familiarity may come at the cost of poorer image-level representation. Thus, if differences between image-level representation of familiar and unfamiliar faces do exist in working memory, then it is most likely that we would observe this cost in the dual-target search condition.

\section{Method}

Participants. Seventy-seven undergraduate students from the University of New South Wales (45 female, mean age $19.1, S D=1.8$ ) took part in the experiment in exchange for course credit. Of these, 21 did not meet the criteria for inclusion. This left a final sample 
of 56 undergraduate students from the University of New South Wales (32 female, mean age $19.2, S D=2.0$ ) took part in the experiment in exchange for course credit.

Stimuli and Procedure. We used the same stimuli and pre-screening questionnaire as in previous experiments. Only participants familiar with at least 10 celebrities and unfamiliar with at least 10 celebrities were included in the study, meaning that 21 participants who completed the study were not included in the final analysis. The remaining participants were on average familiar with $65 \%$ of the 'familiar' celebrities and $3 \%$ of 'unfamiliar' celebrities.

On each trial, participants were first presented with either one or two target images on screen for either 4 or 8 seconds respectively. After being shown the target image/s, participants were presented with the search array that consisted of 48 images of that same identity (Figure 7). Of these, 8 images were duplicates of the target image(s), with the remainder being 40 other images of that same person. When participants had to search for 2 targets, 4 duplicates of each image were present in the array. Distractor images also occurred 4 or 8 times in the array in order to ensure the target did not stand out as the only duplicate image. Participants had to complete an "exhaustive search" on each array (Biggs, 2017), and were instructed to select the target images by clicking on them until all copies of the target image(s) were found. If the response was incorrect, participants were given feedback on screen, as well as a 4-second "time out" penalty. Participants continued responding by clicking on images until either all the targets were found or 6 consecutive incorrect selections were made.

Participants completed 2 practice trials before attempting the main task, which 20 trials split into 4 blocks of equal length. Block order was randomised for each participant. Both the number of search targets and the familiarity of the target and distractors remained the same within a block. 


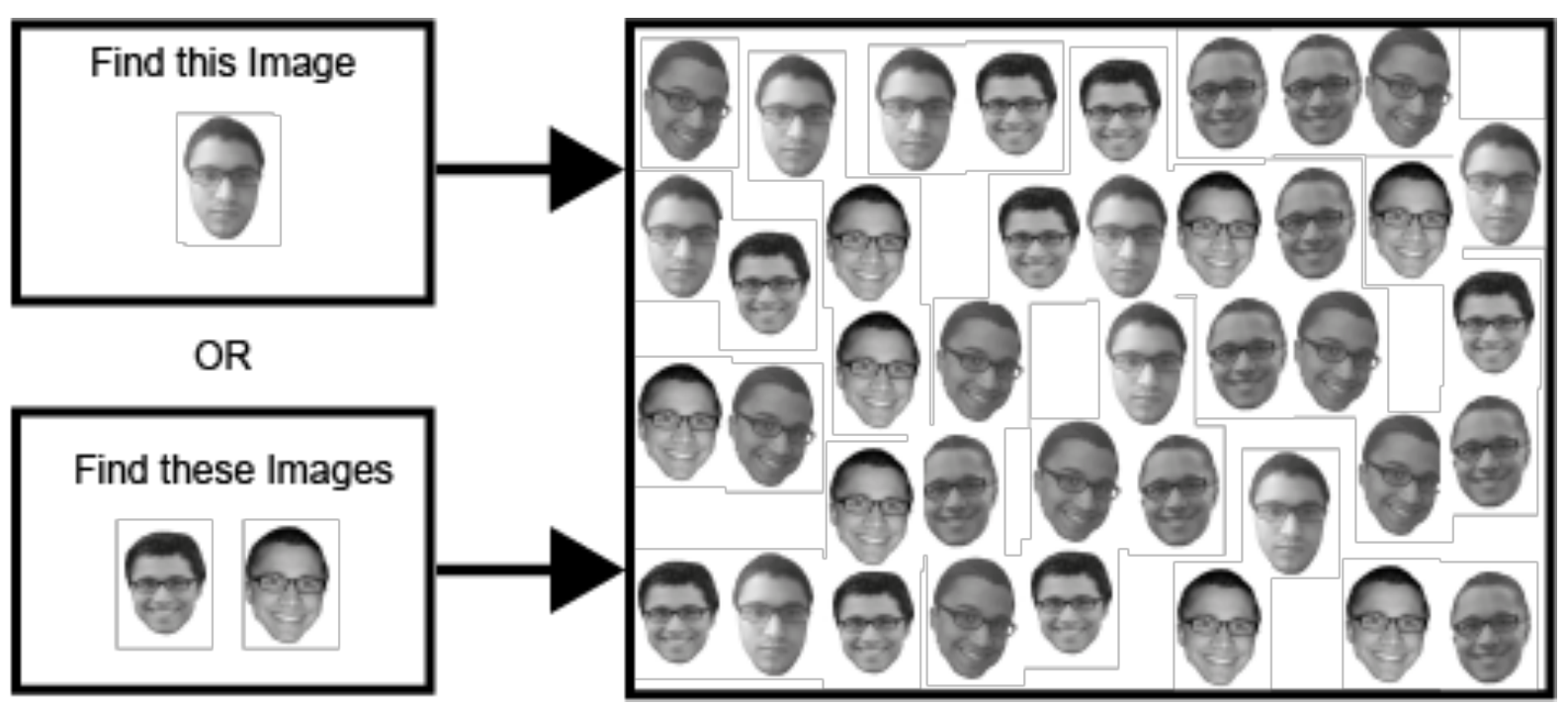

Figure 7. Visual search paradigm used in Experiment 4. Participants were shown target image (top left panel) or images (bottom left panel), which they were required to locate in a search array (right panel). The target image(s) occurred eight times in the search array.

The target images disappeared as participants clicked on them in the test array. The trial ended when either all targets were found or six consecutive errors were made.

\section{Results}

As this study required exhaustive search, accuracy was calculated by computing the mean error rate of each participant across trials: Defined as the number of incorrect selections made divided by the total number of selections. This provided us with a percentage score with higher values indicating more errors being made. We also compared the mean trial length across conditions, defined as the average time spent on each array until either all the targets were found or the maximum number of consecutive errors (6) was reached. These performance data are shown in Figure 8. We analysed error rates and trial length separately in $2 \times 2$ repeated measures ANOVA with Familiarity (familiar, unfamiliar) and Number of Targets (one, two) as factors.

Accuracy. For percentage errors, the main effect of Familiarity was non-significant, $F(1,55)=0.32, p=.572, \eta_{\mathrm{p}}^{2}=.006$. The main effect of Number of Targets was significant, 
with significantly more errors being made when participants had to search for two targets compared to one, $F(1,55)=122.52, p<.001, \eta_{\mathrm{p}}{ }^{2}=.690$. The interaction between factors was non-significant, $F(1,55)=3.28, p=.076, \eta_{\mathrm{p}}{ }^{2}=.056$. For the critical main effect of Familiarity, estimated Bayes factor show the data were 6.51 times more likely to occur in a model without familiarity as a factor, again providing support for the null hypothesis.
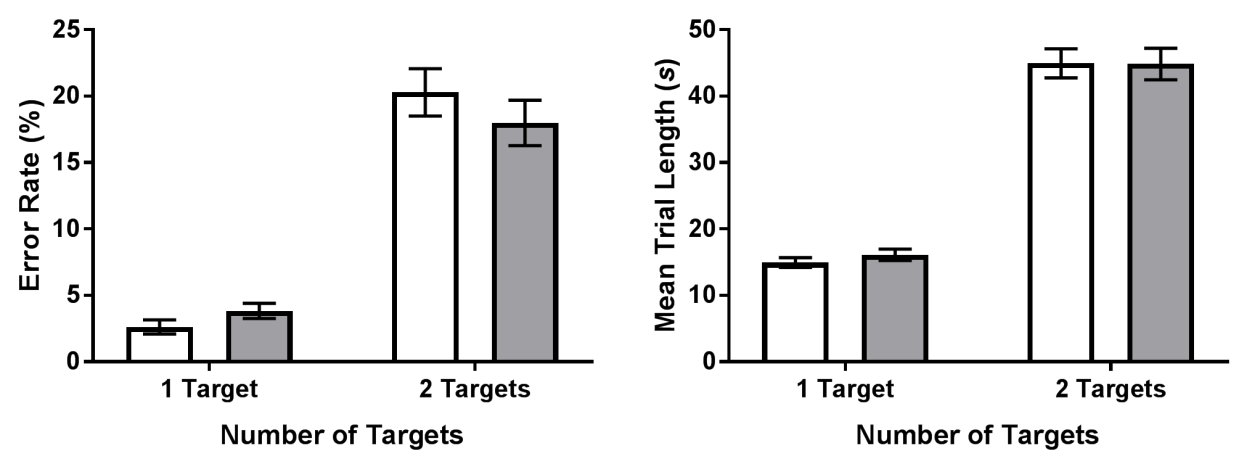

口 Unfamiliar
口 Familiar

Figure 8. Performance in the visual search paradigm employed in Experiment 4.

Error bars denote \pm 1 standard error.

Trial length. For mean trial duration, there was a non-significant main effect of Familiarity, $F(1,55)=0.14, p=.710, \eta_{\mathrm{p}}^{2}=.003$. The main effect of Number of Targets was significant, with participants being slower with two targets than compared to one $F(1,55)=$ $340.70, p<.001, \eta_{\mathrm{p}}^{2}=.861$. The interaction between factors was non-significant, $F(1,55)=$ $0.20, p=.659, \eta_{\mathrm{p}}{ }^{2}=.004$. For the critical main effect of Familiarity, the estimated Bayes factor suggested the data were 6.93 times more likely to occur in a model without familiarity as a factor than in a model with this factor.

\section{Discussion}

Experiment 4 investigated whether familiarity affected performance on a challenging visual search task that required participants to find a particular image when the distractors were other images of the same person. Again, we found that there were no overall differences 
in performance between unfamiliar and familiar faces. We did observe very substantial costs for searching for two images of a face, which were comparable to the costs observed when searching for two different unfamiliar faces (Mestry et al., 2017). However, these dual-target costs were equivalent for familiar and unfamiliar faces, suggesting that familiarity does not affect the image-specificity of representations driving visual search performance on this task.

\section{Experiment 5}

In the previous four experiments, we found consistent support for the null hypothesis that familiarity does not affect the retention of image-specific information in short term memory. However, in all of these experiments, participants were given explicit instructions to remember the precise images shown to them in the study phase. This instruction may have led to participants overriding normal encoding processes to encode pictorial details of familiar face images. Supporting this, Armann et al. (2016) reported modulation of response behaviour when participants were instructed to remember the precise details of an image versus when they were instructed to remember the person in the image.

In Experiment 5, we test whether the null effect of familiarity can be explained by the instructions to remember the precise image, or whether null effects are also observed when participants do not know whether to encode image or identity information. Participants viewed unfamiliar or familiar faces and had to either make an image-based judgement, i.e. "did you see this image before?”, or an identity-based judgment, i.e. "did you see this person before?". Critically, we compared performance when these instructions were provided before encoding the images to when trial instructions were only provided after encoding the target images (c.f. Yan et al. 2017). If encoding of image-specific details were modulated by task instruction, then we would expect differences in performance between these conditions.

We also addressed some potential limitations of previous experiments. Participants in Experiments 1 to 4 were not typically very familiar with the Australian local celebrities, and 
so it is possible that low levels of familiarity drove null effects. This also led to a relatively small number of trials per condition. Here, we use 'a-list' international celebrities that were chosen to be highly familiar to Australian undergraduate students to address these considerations.

\section{Method}

Participants. Sixty-nine undergraduate students from the University of New South Wales (58 female, mean age 20.2 SD =4.6) took part in the experiment in exchange for course credit. Of these 69 students, 17 did not meet the criteria for inclusion based on familiarity and 1 failed to perform correctly on the catch trials (see Design and Procedure). This left a final sample of 51 participants (44 female, mean age 20.4, $S D=5.1$ ).

Stimuli. Australian celebrities that were used as the 'Familiar' faces in previous experiments were replaced by 32 international celebrities, who were selected due to their popularity in mainstream pop culture. Further, from the UK celebrities set we selected the 32 celebrities who were least often recognised by Australian participants to be used as the unfamiliar face in this task. For each identity, we obtained 20 unique images from Google Image Search. Unlike in previous experiments we did not remove the backgrounds of these images, for consistency with the images used by Armann et al (2016). Images were cropped using a rectangular marquee with a 3:4 aspect ratio and rescaled to $225 \times 300$ pixels.

Design and procedure. The trial structure for this experiment is illustrated in Figure 9. On each trial, participants were required to make either an image-based decision (" did you see this image before?") or an identity-based decision ("did you see this person before?"). On the first screen participants were either given the instruction for that trial before viewing the target images (Before condition), or shown no instructions on the first screen (After 
condition). This instruction was a single word prompt and the meaning of this prompt was explained prior to beginning the experiment. Following this, an array of 4 images of different people were shown on screen for 4 seconds. Participants were then shown a central fixation cross for 0.5 seconds followed by a visual mask for 1 second. In both conditions, participants were then given the task instruction (image of identity) before the probe image was presented for 3 seconds, and then had to decide if the probe was a match or non-match. Participants were instructed to respond as quickly and as accurately as possible on this final screen.

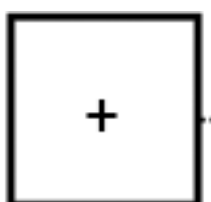

0.5 seconds

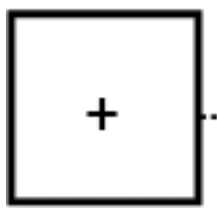

0.5 seconds

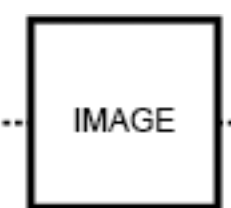

3 seconds

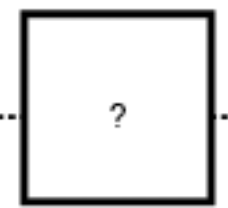

3 seconds

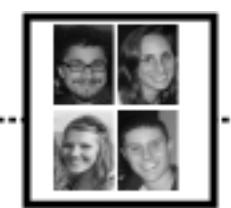

4 seconds

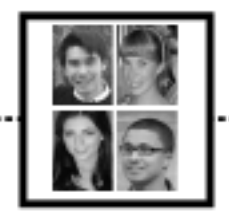

4 seconds

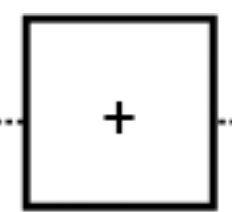

0.5 seconds

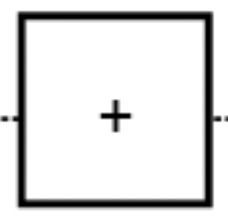

0.5 seconds

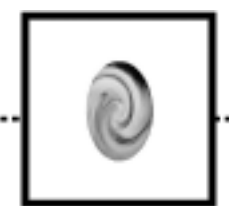

1 second

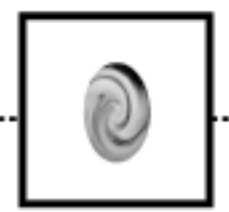

1 second

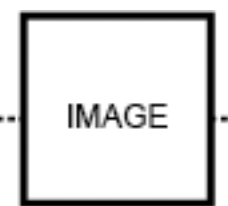

3 seconds

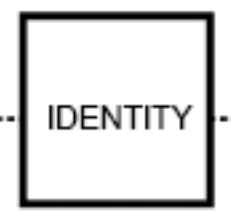

3 seconds

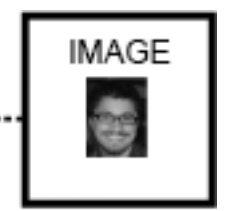

Until response

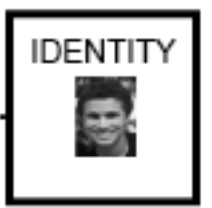

Until response

Figure 9. The image and identity memory paradigm employed used in Experiment 4.

Participants were shown 4 target images of either unfamiliar or familiar faces and had to make either an image (did you see this image before?) or identity (did you see this person before?) decision. The trial instruction was either given before (top panel) or after (bottom panel) viewing the target images (correct responses are top panel: "non-match", bottom panel: “match”).

Participants completed 4 practice trials before attempting the main task, which consisted of 165 trials split into 5 blocks of equal length. Block order was randomised for each participant. Each block contained the same number of trials of each condition presented in a random order and equal numbers of match and non-match trials.

Because we expected participants to be highly familiar with the celebrities used in this 
experiment, all UK celebrities were included in analysis as unfamiliar faces, and all international celebrities were included as familiar faces. As a result, we used a stricter criterion to select eligible participants for analysis: Only participants familiar with more than $80 \%$ of the international celebrities and familiar with less than $20 \%$ of the UK celebrities were included in the final analysis. This criterion resulted in 18 participants who completed the study being excluded prior to analysis. The remaining participants were on average familiar with $97 \%$ of the international celebrities and $5 \%$ of UK celebrities.

\section{Results}

Sensitivity and criterion scores are shown in Figure 10. These data were analysed separately using a $2 \times 2 \times 2$ repeated measures ANOVA with factors Familiarity (familiar, unfamiliar), Trial Type (image, identity) and Instruction Timing (before, after).
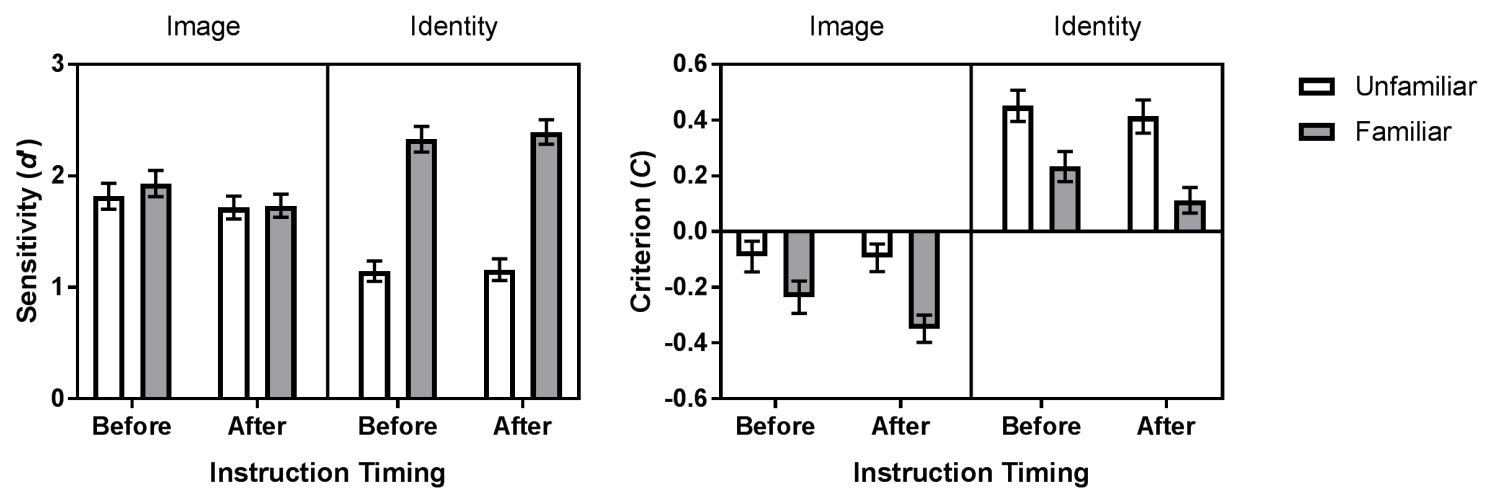

Figure 10. Performance on memory paradigm. Sensitivity (d') and response criterion (C) for Experiment 5. Error bas denote \pm 1 standard error.

Sensitivity. For sensitivity scores, the main effect of Familiarity was significant, $F(1$, $50)=98.344, p<.001, \eta_{\mathrm{p}}{ }^{2}=.663$, as participants had higher sensitivity overall with familiar faces than unfamiliar faces. Critically, this effect was qualified by a significant interaction between Familiarity and Trial Type, $F(1,50)=93.52, p<.001, \eta_{\mathrm{p}}{ }^{2}=.655$. Follow up comparisons show significantly higher sensitivity for familiar faces than unfamiliar faces on 
the identity trials, $t(50)=12.635, p<.001$, Cohen's $d=3.579$, but no significant differences between the familiar and unfamiliar faces on the image trials, $t(50)=0.826, p=.413$, Cohen's $d=0.230$. This interaction was also examined by estimating a Bayes factor for each pairwise comparison. For the identity trials, the Bayes factor suggests that the data was 505,679 times more likely to occur in a model with familiarity as a factor than one without it. Conversely, for the image trials the Bayes factor suggests that the data was 3.86 times more likely to occur in model without familiarity as a factor than one with it. All other main effects and interactions were not significant, Trial Type: $F(1,50)=0.347, p=.559, \eta_{\mathrm{p}}{ }^{2}=.007$; Instruction Timing: $F(1,50)=0.913, p=.344, \eta_{\mathrm{p}}{ }^{2}=.018$; Familiarity*Instruction Timing: $F(1,50)=0.033, p=.856, \eta_{\mathrm{p}}{ }^{2}=.001$; Trial Type*Instruction Timing: $F(1,50)=2.966, p=$ $.091, \eta_{\mathrm{p}}{ }^{2}=.056$; three-way interaction: $F(1,50)=0.496, p=.485, \eta_{\mathrm{p}}^{2}=.010$.

Criterion. For criterion scores, the main effect of Familiarity was significant, $F(1,50)$ $=50.532, p<.001, \eta_{\mathrm{p}}^{2}=.503$, with participants showing a greater bias to say "Non-match" for unfamiliar faces than familiar faces. There was a significant main effect of Trial Type, $F(1,50)=111.397, p<.001, \eta_{\mathrm{p}}^{2}=.690$, with participants showing a tendency to say "Match" more in the image trials and a tendency to say "Non-match" more in the identity trials. The main effect of Instructions was also significant, $F(1,50)=7.278, p=.009, \eta_{\mathrm{p}}{ }^{2}=.127$, with participants on average saying "Non-match" more when the trial instructions were shown before the target screen than when instructions were only shown after the target screen. However, all of the interactions between factors were not significant, Familiarity*Trial Type: $F(1,50)=1.112, p=.297, \eta_{\mathrm{p}}^{2}=.022 ;$ Familiarity*Instruction Timing: $F(1,50)=3.751, p=$ $.058, \eta_{\mathrm{p}}{ }^{2}=.070$; Trial Type*Instruction Timing: $F(1,50)=0.180, p=.673, \eta_{\mathrm{p}}{ }^{2}=.004$; threeway interaction: $F(1,50)=0.052, p=.821, \eta_{\mathrm{p}}^{2}=.001$. 


\section{Discussion}

The results of Experiment 5 suggest that task instructions cannot account for null effects of familiarity in previous experiments. Thus, it is likely that image-specific information is encoded in short-term memory to an equivalent extent for both familiar and unfamiliar faces. Further, this encoding would appear to be relatively automatic, as it is not affected by task instructions.

\section{Meta-Analysis of Experiments 1, 2, 3 \& 5}

Armann et al. (2016) report that familiarity causes a disadvantage in long-term memory for image-specific information. Building on this work, we aimed to quantify support for the hypothesis that sensitivity in short-term image memory tasks is also reduced for familiar faces (i.e. one-tailed hypothesis, $\mathrm{H}_{1}: \mu_{1}>\mu_{2}$ ). Across the five experiments reported in this paper, we found consistent support in favour of the null hypothesis: That familiarity does not modulate encoding of image-specific details in memory. To formalise the level of support across these experiments we performed a meta-analysis of data from Experiments 1, 2, 3, and

5. Given that these four experiments examined the same hypothesis and standardised measures of d-prime, we were able to examine the cumulative evidence across these experiments. Experiment 4 did not use signal detection measures and so we excluded this dataset from the analysis.

Figure 11 shows the cumulative support for the null hypothesis as each of the 214 participants was added to the analysis. The overall Bayes factor was 31.7 in favour of the null, meaning that the data observed across Experiments 1, 2, 3, and 5 were 31.7 times more likely to have occurred in the case that sensitivity for unfamiliar faces is not higher than for familiar faces, relative to in a case where it was. Visual inspection of figure also shows that support in favour of the null hypothesis stabilised at a Bayes Factor of 30 as $\mathrm{n}$ approached 100 participants, with little change thereafter. Overall, these results provide very strong 
support for the conclusion that sensitivity to image-level details was not reduced when participants were familiar with the identity depicted in the image.

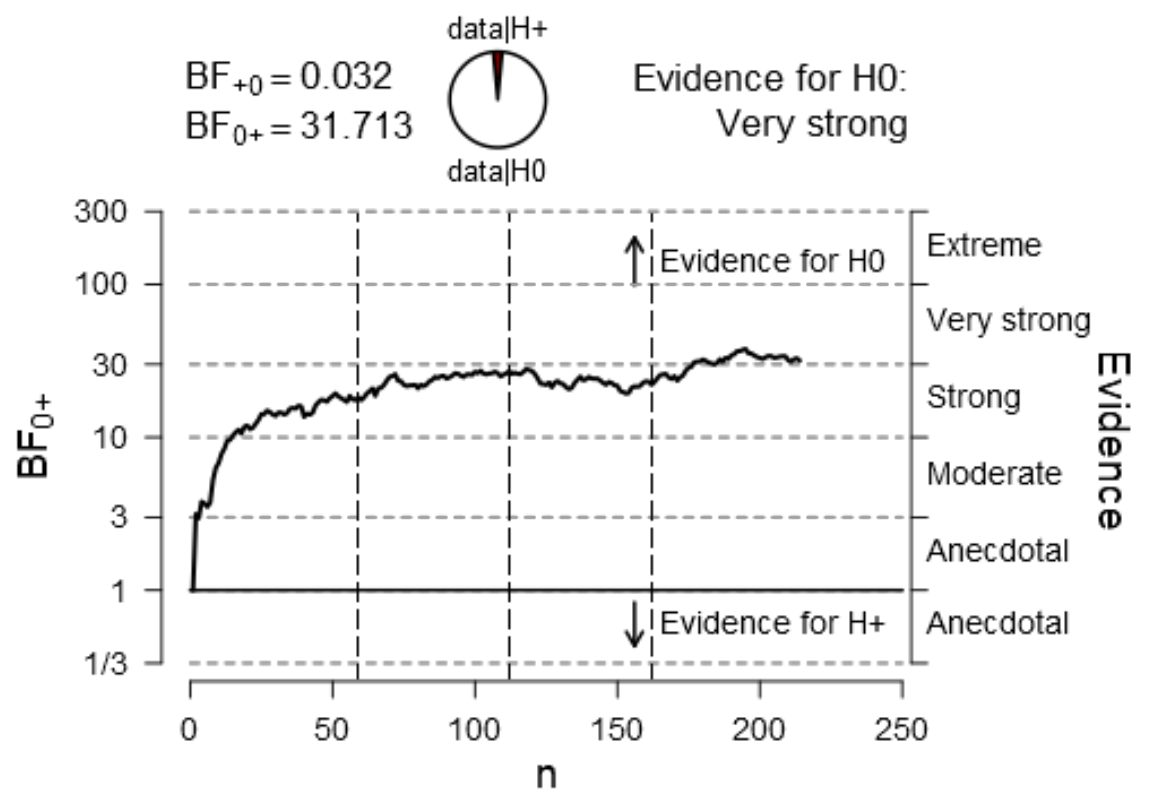

Figure 11. Accumulated evidence for/against the one-tailed hypothesis that sensitivity is greater for unfamiliar faces than familiar faces for all participants in Experiments 1, 2, 3, and 5. Bayes factor is calculated as each of the 214 participants is added to the analysis, in chronological order of participants tested, with vertical dashed lines segmenting cohorts from the four experiments. Further details of this analysis are provided in main text.

\section{General Discussion}

In five experiments, we examined performance on tasks that required participants to retain image-level details of face images in visual working memory. These experiments were designed to test whether image-specific details are encoded in memory for familiar and unfamiliar faces to the same extent. This is of critical importance to understanding how longterm memory representations of familiar faces interact with early memory encoding processes, because a potential route to forming image-invariant representations of familiar 
faces is via perceptual and memory encoding processes that inhibit the storage of transient image-level details.

Importantly, the different experimental paradigms selected in this paper have all shown an advantage for familiar over unfamiliar faces in versions that required participants to discriminate identity. Familiarity is known to benefit tasks that require matching face identity across short memory retention intervals, for example face detection (Visconti di Oleggio Castello \& Gobbini, 2015), face matching (Bruce et al., 2001; Megreya \& Burton, 2007), change detection (Jackson \& Raymond, 2008), and visual search (Dunn et al., 2018; Ito \& Sakurai, 2014; Tong \& Nakayama, 1999). Despite this, across all experiments we found no differences between memory for specific images of unfamiliar and familiar faces. Together, these results provide strong evidence that familiarity does not affect the encoding of imagespecific details in memory.

This places important constraints on models of face processing where hierarchical representations progressively abstract visual information from the input image. An early example of this architecture is the distinction between pictorial and structural descriptions proposed by Vicki Bruce and Andy Young (Bruce \& Young, 1986; see also Haxby, Hoffman \& Gobbini, 2000; Gobbini \& Haxby, 2007). This model proposes that when faces are encountered by the visual system, they are represented at a pictorial level that retains information specific to a given photograph, and at a higher level of abstraction that encodes structural properties of the face, before being matched to long-term memory representations of familiar faces (i.e. Face Recognition Units; see Bruce \& Young, 1986; Burton \& Bruce, 1993). Structural descriptions are abstracted from the pictorial information, to enable accurate matching to long-term representations by capturing invariant aspects of a face that distinguish it from other faces. 
The Bruce and Young (1986) framework does not specify the nature of interactions between pictorial, structural and long-term representations. However, more recent neurological models retain these distinctions and specify bi-directional links between higherlevel processing stages - that instantiate view invariant representations of familiar faces - and early visual areas that represent the retinal images (e.g. Haxby et al. 2000, Duchaine \& Yovel, 2015). To shed more light on this bidirectional relationship, the comparison between familiar and unfamiliar face image memory in this paper was a test of whether pictorial details retained in early stages of memory storage are affected by top-down influences from familiar face representations. Our results show that pictorial details are encoded in short-term memory to an equal extent for familiar and unfamiliar faces, and so we conclude that differences in memory for pictorial information (Armann et al, 2016) are not due to initial encoding processes.

Our results also constrain future theoretical accounts of face processing that aim to incorporate predictive coding frameworks (Trapp et al., 2018). Predictive coding theory proposes that sensory data are constrained by top-down predictions based on contextual information and the learning of stimulus properties (e.g. Rao \& Ballard, 1999; see Apps \& Tsakiris, 2013). Effects of long-term learning of stimulus properties have been shown to have strong effects on early perceptual processing (see Gregory, 2005). In support of the possibility that memory representations for familiar faces also exert such an influence, the earliest face-specific electrophysiological markers show sensitivity to expectancies about the identity that is being perceived (N170: Johnston et al. 2016). Further, fMRI studies show that neuronal representations in areas associated with early processing of faces (OFA) differ for familiar and unfamiliar faces (Natu \& O’Toole, 2015; cf. Ewbank et al. 2013).

Thus, stored representation of faces may plausibly exert a top-down influence on the encoding of face images. This prediction was also inspired by a recent study by Armann et al. 
(2016) that examined whether long-term memory for specific images of faces - measured using a standard recognition memory paradigm - is affected by face familiarity. They found that participants were less accurate when recognising studied images of familiar faces, leading the authors to infer that "unfamiliar faces are encoded more pictorially and familiar faces more abstractly” (p. 575). Importantly however, Armann et al's (2016) study did not identify the stage of processing that gives rise to this qualitative difference. Our results show that the cost of familiarity reported by Armann et al (2016) is not caused by reduced encoding of pictorial information for familiar faces. Instead, it appears that the cost occurs in longerterm memory storage.

This result is consistent with previous studies showing that image-specific details are encoded in representations of familiar faces. For example, a number of studies have shown that repetition priming for familiar faces benefits from repetition of the same image relative to a different image (e.g. Ellis, Flude, Young \& Burton, 1996), suggesting that pictorial details are encoded in memory to some extent. Similarly, Ritchie, Kramer, and Burton (2018) recently found that averages of familiar faces - which retain invariant features but not imagespecific details - were rated as a poorer likeness than individual images. Instead, they found there was an advantage for photos that appeared to be particularly 'iconic' representations (cf. Carbon, 2008). In addition, Chapman and Susilo (2018) show that face recognition across both short and long retention intervals is impaired by changing the image between learning and test exposures, but that this impairment is equivalent for familiar and unfamiliar faces.

Our results therefore combine with previous studies to emphasise the importance of pictorial codes in familiar face perception (Weibert, Flack, Young, \& Andrews, 2018) and face learning (Longmore, Liu \& Young, 2008). As these authors have proposed, the function of pictorial representation in face perception may be to provide a common trace that can be processed by different branches of the face processing network; to enable, for example, 
parallel processing of expression and identity (Weibert et al. 2018). Consistent with this proposal, results of Experiment 5 show that the storage of these details was unaffected by task instructions, and so the retention of pictorial details in memory appears to be a relatively mandatory process (see also Yan et al. 2017).

In light of our findings, it will be important in future work to discriminate between competing accounts of why costs of familiarity reported by Armann et al. (2016) arise in long-term memory. One possibility is that familiarity modulates the processing of intermediate representations that channel information to long-term memory representations (e.g. a 'structural description'), and that pictorial information is lost during formation of these descriptions.

Alternatively, it could be that pictorial information is passed to long-term memory for both familiar and unfamiliar faces but that pictorial information is less accessible for familiar faces. For example, the more one becomes familiar with a face, the more resource competition there will be for representing specific views of that face, which may lead to impaired accuracy when discriminating between new and previously encountered images of a familiar face (see Perrett, Oram, \& Ashbridge, 1998). Importantly, this latter account does not entail any qualitative difference in processing of pictorial information for familiar and unfamiliar faces, but rather the cost of familiarity reported by Armann et al. (2016) may arise naturally from quantitative differences (i.e. in the number of instances that are encoded in memory).

In summary, we show that top-down influence of familiarity on pictorial information does not influence retention of image specific details in early stages of memory encoding. This may reflect the independence of representations that encode pictorial and more abstract properties responsible for face recognition (see Henson, Shallice \& Dolan, 2000). Our results underline the pervasiveness of image-specific details in representation of familiar faces, but 
the extent to which image-specific details are retained at each stage in the face processing system remains unclear. Future work is therefore necessary to characterise the relationship between pictorial and abstractive representation in the face processing system.

\section{References}

Apps, M. A., \& Tsakiris, M. (2013). Predictive codes of familiarity and context during the perceptual learning of facial identities. Nature communications, 4, 2698. https://doi.org/10.1038/ncomms3698

Armann, R. G., Jenkins, R., \& Burton, A. M. (2016). A familiarity disadvantage for remembering specific images of faces. J Exp Psychol Hum Percept Perform, 42(4), 571-580. https://doi.org/10.1037/xhp0000174

Balas, B., Cox, D., \& Conwell, E. (2007). The effect of real-world personal familiarity on the speed of face information processing. PLoS One, 2(11), e1223. https://doi.org/10.1371/journal.pone.0001223

Balas, B., \& Pearson, H. (2017). Intra- and extra-personal variability in person recognition. Visual Cognition, 25(4-6), 456-469. https://doi.org/10.1080/13506285.2016.1274809

Baker, K. A., Laurence, S., \& Mondloch, C. J. (2017). How does a newly encountered face become familiar? The effect of within-person variability on adults' and children's perception of identity. Cognition, 161, 19-30.

Biggs, A. T. (2017). Getting satisfied with "satisfaction of search": How to measure errors during multiple-target visual search. Atten Percept Psychophys. https://doi.org/10.3758/s13414-017-1300-2

Brainard, D. H. (1997). The Psychophysics Toolbox. Spat Vis, 10(4), 433-436. https://doi.org/10.1163/156856897X00357 
Bravo, M. J., \& Farid, H. (2009). The specificity of the search template. J Vis, 9(1), 34 31-39. https://doi.org/10.1167/9.1.34

Bravo, M. J., \& Farid, H. (2012). Task demands determine the specificity of the search template. Atten Percept Psychophys, 74(1), 124-131. https://doi.org/10.3758/s13414$\underline{011-0224-5}$

Bruce, V. (1982). Changing faces: Visual and non-visual coding processes in face recognition. Br J Psychol, 73, 105-116. https://doi.org/10.1111/j.2044$\underline{\text { 8295.1982.tb01795.x }}$

Bruce, V. (1994). Stability from variation: the case of face recognition. The M.D. Vernon Memorial Lecture. Q J Exp Psychol A, 47(1), 5-28. https://doi.org/10.1080/14640749408401141

Bruce, V., Henderson, Z., Greenwood, K., Hancock, P. J. B., Burton, A. M., \& Miller, P. (1999). Verification of face identities from images captured on video. Journal of Experimental Psychology-Applied, 5(4), 339-360. https://doi.org/10.1037//1076$\underline{898 \times .5 .4 .339}$

Bruce, V., Henderson, Z., Newman, C., \& Burton, A. M. (2001). Matching identities of familiar and unfamiliar faces caught on CCTV images. J Exp Psychol Appl, 7(3), 207218. http://doi.org/10.1037/1076-898X.7.3.207

Bruce, V., \& Young, A. (1986). Understanding face recognition. Br J Psychol, 77 ( Pt 3), 305-327. https://doi.org/10.1111/j.2044-8295.1986.tb02199.x

Burton, A. M., \& Bruce, V. (1993). Naming faces and naming names: exploring an interactive activation model of person recognition. Memory, 1(4), 457-480. https://doi.org/10.1080/09658219308258248 
Burton, A. M., Jenkins, R., Hancock, P. J., \& White, D. (2005). Robust representations for face recognition: The power of averages. Cogn Psychol, 51(3), 256-284. https://doi.org/10.1016/j.cogpsych.2005.06.003

Burton, A. M., Kramer, R. S., Ritchie, K. L., \& Jenkins, R. (2016). Identity From Variation: Representations of Faces Derived From Multiple Instances. Cogn Sci, 40(1), 202-223. https://doi.org/10.1111/cogs.12231

Burton, A. M., White, D., \& McNeill, A. (2010). The Glasgow Face Matching Test. Behav Res Methods, 42(1), 286-291. https://doi.org/10.3758/BRM.42.1.286

Burton, A. M., Wilson, S., Cowan, M., \& Bruce, V. (1999). Face recognition in poor-quality video: Evidence from security surveillance. Psychol Sci, 10(3), 243-248. https://doi.org/10.1111/1467-9280.00144

Caharel, S., Ramon, M., \& Rossion, B. (2014). Face familiarity decisions take $200 \mathrm{msec}$ in the human brain: Electrophysiological evidence from a go/no-go speeded task. Journal of Cognitive Neuroscience, 26(1), 81-95. https://doi.org/10.1162/jocn_a_00451

Calder, A. J., \& Young, A. W. (2005). Understanding the recognition of facial identity and facial expression. Nat Rev Neurosci, 6(8), 641-651. https://doi.org/10.1038/nrn1724

Carbon, C. C. (2008). Famous faces as icons. The illusion of being an expert in the recognition of famous faces. Perception, 37(5), 801-806.

https://doi.org/10.1068/p5789

Chapman, A., \& Susilo, T. (2018). How robust is familiar face recognition? A repeat detectionstudy of >1,000 photographs. Royal Society Open Science, 5, 170634. https://doi.org/10.1098/rsos.170634 
Collins, E., Robinson, A. K., \& Behrmann, M. (2018). Distinct neural processes for the perception of familiar versus unfamiliar faces along the visual hierarchy revealed by EEG. NeuroImage, 181, 120-131.

Duchaine, B., \& Yovel, G. (2015). A revised neural framework for face processing. Annual Review of Vision Science, 1, 393-416.

Dunn, J. D., Kemp, R. I., \& White, D. (2018). Search templates that incorporate within-face variation improve visual search fr faces. Cognitive Research: Principles and Implications, 3, 37. https://doi.org/10.1186/s41235-018-0128-1

Ellis, A. W., Flude, B. M., Young, A., \& Burton, A. M. (1996). Two loci of repetition priming in the recognition of familiar faces. Journal of Experimental Psychology: Learning, Memory, and Cognition, 22(2), 295. http://doi.org/10.1037/0278$\underline{7393.22 .2 .295}$

Erdfelder, E., Faul, F., \& Buchner, A. (1996). GPOWER: A general power analysis program. Behavior Research Methods, Instruments, Computers, 28(1), 1-11. https://doi.org/10.3758/BF03203630

Ewbank, M. P., Henson, R. N., Rowe, J. B., Stoyanova, R. S., \& Calder, A. J. (2013). Different neural mechanisms within occipitotemporal cortex underlie repetition suppression across same and different-size faces. Cerebral Cortex, 23(5), 1073-1084. https://doi.org/10.1093/cercor/bhs070

Gobbini, M. I., \& Haxby, J. V. (2007). Neural systems for recognition of familiar faces. Neuropsychologia, 45(1), 32-41. https://doi.org/10.1016/j.neuropsychologia.2006.04.015

Gregory, R. L. (2005). The Medawar lecture 2001 knowledge for vision: Vision for knowledge. Philosophical Transactions of the Royal Society of London B: Biological Sciences, 360(1458), 1231-1251. https://doi.org/10.1098/rstb.2005.1662 
Haxby, J. V., Hoffman, E. A., \& Gobbini, M. I. (2000). The distributed human neural system for face perception. Trends in Cognitive Sciences, 4(6), 223-233. https://doi.org/10.1016/S1364-6613(00)01482-0

Henson, R., Shallice, T., \& Dolan, R. (2000). Neuroimaging evidence for dissociable forms of repetition priming. Science, 287(5456), 1269-1272. https://doi.org/10.1126/science.287.5456.1269

Hout, M. C., \& Goldinger, S. D. (2015). Target templates: the precision of mental representations affects attentional guidance and decision-making in visual search. Atten Percept Psychophys, 77(1), 128-149. https://doi.org/10.3758/s13414-014-0764$\underline{6}$

Ito, H., \& Sakurai, A. (2014). Familiar and Unfamiliar Face Recognition in a Crowd. Psychology, 05(09), 1011-1018. https://doi.org/10.4236/psych.2014.59113

Jackson, M. C., \& Raymond, J. E. (2008). Familiarity enhances visual working memory for faces. J Exp Psychol Hum Percept Perform, 34(3), 556-568. https://doi.org/10.1037/0096-1523.34.3.556

Jenkins, R., White, D., Van Montfort, X., \& Burton, A. M. (2011). Variability in photos of the same face. Cognition, 121(3), 313-323. https://doi.org/10.1016/j.cognition.2011.08.001

Johnston, P., Overell, A., Kaufman, J., Robinson, J., \& Young, A. W. (2016). Expectations about person identity modulate the face-sensitive N170. Cortex, 85, 54-64. https://doi.org/10.1016/j.cortex.2016.10.002

Johnston, R. A., \& Edmonds, A. J. (2009). Familiar and unfamiliar face recognition: a review. Memory, 17(5), 577-596. https://doi.org/10.1080/09658210902976969 
Longmore, C. A., Liu, C. H., \& Young, A. W. (2008). Learning faces from photographs. $J$ Exp Psychol Hum Percept Perform, 34(1), 77-100. https://doi.org/10.1037/0096$\underline{1523.34 .1 .77}$

Megreya, A. M., \& Burton, A. M. (2006). Unfamiliar faces are not faces: Evidence from a matching task. Mem Cognit, 34(4), 865-876. https://doi.org/10.3758/BF03193433

Megreya, A. M., \& Burton, A. M. (2007). Hits and false positives in face matching: a familiarity-based dissociation. Percept Psychophys, 69(7), 1175-1184. https://doi.org/10.3758/BF03193954

Megreya, A. M., \& Burton, A. M. (2008). Matching faces to photographs: poor performance in eyewitness memory (without the memory). J Exp Psychol Appl, 14(4), 364-372. https://doi.org/10.1037/a0013464

Mestry, N., Menneer, T., Cave, K. R., Godwin, H. J., \& Donnelly, N. (2017). Dual-Target Cost in Visual Search for Multiple Unfamiliar Faces. J Exp Psychol Hum Percept Perform. https://doi.org/10.1037/xhp0000388

Natu, V. \& O’Toole, A.J. (2015). Spatiotemporal changes in neural response patterns to faces varying in visual familiarity. NeuroImage. 108, 151-159. http://doi.org/10.1016/j.neuroimage.2014.12.027

Perrett, D. I., Oram, M. W., \& Ashbridge. (1998). Evidence accumulation in cell populations responsive to faces: an account of generalisation of recognition without mental transformations. Cognition, 67, 111-145. http://doi.org/10.1016/S0010$\underline{0277(98) 00015-8}$

Ramon, M., \& Gobbini, M. I. (2018). Familiarity matters: A review on prioritized processing of personally familiar faces. Visual Cognition, 26(3), 179-195. https://doi.org/10.1080/13506285.2017.1405134 
Rao, R. P., \& Ballard, D. H. (1999). Predictive coding in the visual cortex: a functional interpretation of some extra-classical receptive-field effects. Nature neuroscience, 2(1), 79. https://doi.org/10.1038/4580

Ritchie, K. L., Kramer, R. S. S., \& Burton, A. M. (2018). What makes a face photo a 'good likeness'? Cognition, 170, 1-8. https://doi.org/10.1016/j.cognition.2017.09.001

Ritchie, K. L., Smith, F. G., Jenkins, R., Bindemann, M., White, D., \& Burton, A. M. (2015). Viewers base estimates of face matching accuracy on their own familiarity: Explaining the photo-ID paradox. Cognition, 141, 161-169. https://doi.org/10.1016/j.cognition.2015.05.002

Rossion, B. (2002). Is sex categorization from faces really parallel to face recognition? Visual Cognition, 9(8), 1003-1020. https://doi.org/10.1080/13506280143000485

Shiffrin, R. M., \& Schneider, W. (1977). Controlled and automatic human information processing: II. Perceptual learning, automatic attending, and a general theory. Psychological review, 84, 127-190. http://doi.org/10.1037/0033-295X.84.2.127

Stroud, M. J., Menneer, T., Cave, K. R., \& Donnelly, N. (2012). Using the dual-target cost to explore the nature of search target representations. J Exp Psychol Hum Percept Perform, 38(1), 113-122. http://doi.org/10.1037/a0025887

Tong, F., \& Nakayama, K. (1999). Robust representations for faces: evidence from visual search. J Exp Psychol Hum Percept Perform, 25(4), 1016-1035. http://doi.org/10.1037/0096-1523.25.4.1016

Trapp, S., Schweinberger, S. R., Hayward, W. G., \& Kovács, G. (2018). Integrating predictive frameworks and cognitive models of face perception. Psychonomic Bulletin \& Review. https://doi.org/10.3758/s13423-018-1433-x 
Visconti di Oleggio Castello, M., Guntupalli, J. S., Yang, H., \& Gobbini, M. I. (2014). Facilitated detection of social cues conveyed by familiar faces. Frontiers in human neuroscience, 8 , 678. https://doi.org/10.3389/fnhum.2014.00678

Visconti di Oleggio Castello, M., \& Gobbini, M. I. (2015). Familiar face detection in 180ms. PloS one, 10(8), e0136548. https://doi.org/10.1371/journal.pone.0136548

Wagenmakers, E. J. (2007). A practical solution to the pervasive problems of $\mathrm{p}$ values. Psychon Bull Rev, 14(5), 779-804. https://doi.org/10.3758/BF03194105

Weibert, K., Flack, T. R., Young, A. W., \& Andrews, T. J. (2018). Patterns Of Neural Response In Face Regions Are Predicted By Low-Level Image Properties. Cortex. https://doi.org/10.1016/j.cortex.2018.03.009

White, D., Burton, A. M., Jenkins, R. \& Kemp, R. I. (2014). Redesigning photo-ID to improve unfamiliar face matching. Journal of Experimental Psychology: Applied, 20 (2), 166-173. https://doi.org/10.1037/xap0000009

White, D., Sutherland, C. A., \& Burton, A. L. (2017). Choosing face: the curse of self in profile image selection. Cognitive research: principles and implications, 2(1), 23. https://doi.org/10.1186/s41235-017-0058-3

Yan, X., Young, A. W., \& Andrews, T. J. (2017). The automaticity of face perception is influenced by familiarity. Atten Percept Psychophys. https://doi.org/10.3758/s13414$\underline{017-1362-1}$ 


\section{Footnotes}

${ }^{1}$ We also conducted a post-hoc analysis of response latencies. Across the five experiments reported in the paper, there was one significant difference between response latency familiar and unfamiliar conditions. This difference was found in Experiment 1, where participants took longer to detect duplicate images of familiar faces relative to unfamiliar faces. These analyses are described in detail in Supplementary Materials.

${ }^{2}$ UK participants were included in Experiment 3 but not in Experiment 2. This was because Experiment 3 was conducted in parallel to Experiment 2, and it became clear after running Experiment 3 participants in the UK that the pattern of data were consistent across UK and Australian participant groups. 


\section{Familiarity does not inhibit image-specific encoding of faces}

Supplementary Analysis

James D. Dunn, Kay L. Ritchie, Richard I. Kemp \& David White

Corresponding author: david.white@unsw.edu.au

\section{Supplementary analysis 1: Median split analysis by participant familiarity}

In all experiments, participants included in the final analysis were familiar with at least 10 of the celebrities that were used as stimuli. However, there was considerable variability in the number of celebrities each participant was familiar with, which may have affected the strength of our familiarity manipulation. To investigate this, we performed a post-hoc 'median split' analysis that tested the effect of familiarity on image memory, separately for 'high familiarity' participants (who were familiar with more celebrities than average) and 'low familiarity participants' (familiar with less than average).

Full details of this analysis are reported below. For each experiment, data were reanalysed separately for high and low familiarity subsamples, using the same ANOVA model reported in the main paper. For brevity, only the critical comparisons of this analysis that involve familiarity are reported.

To summarise, across 5 experiments we did not observe a cost of familiarity in any of the image memory tasks, for either low or high familiarity participants. In Experiment 1, we did find a main effect of familiarity for the 'high familiarity' group but this was in the opposite direction to predicted. Based on this analysis, we conclude that differences in participants' familiarity with the celebrities cannot account for the lack of differences found between unfamiliar and familiar faces.

Experiment 1. Median familiarity with the 'familiar' celebrities was $75 \%$. We reanalysed d-prime scores separately for the high familiarity group $(n=25$, Mean familiarity $=90.1 \%, S D=7.1, \min =77.5 \%, \max =100 \%$ ) and the low familiarity group $(n=31, \mathrm{M}=58.9 \%, S D=14.2, \min =28 \%, \max =75 \%)$. Six participants with the median familiarity score were assigned to low familiarity group.

For the high familiarity group, there was a significant main effect of Familiarity, $F(1$, 
$24)=5.172, p=.032, \eta_{\mathrm{p}}{ }^{2}=.177$, but in to the opposite direction to predicted, with sensitivity being higher for familiar faces on this task than for unfamiliar faces. The interaction between Familiarity and Array Size was not significant, $F(1,24)=0.711, p$ $=.496, \eta_{\mathrm{p}}{ }^{2}=.029$. For the low familiarity group the main effect of Familiarity, $F(1,30)$ $=0.089, p=.767, \eta_{\mathrm{p}}^{2}=.003$, and interaction between factors, $F(1,30)=2.634, p=$ $.080, \eta_{\mathrm{p}}^{2}=.081$, were not significant.

Experiment 2. Median familiarity with 'familiar' celebrities was 53.8\%. Average familiarity for the high familiarity group was $72.9 \%(n=28 ; S D=12.8$, $\min =$ $55 \%, \max =100 \%$ ) and average familiarity for the low familiarity group was $41.5 \%((n=28 ; S D=6.4, \min =32.5 \%, \max =52.5 \%)$.

For the high familiarity group, both the main effect of Familiarity, $F(1,27)=0.184, p=$ $.671, \eta_{\mathrm{p}}{ }^{2}=.007$, and interaction between factors, $F(1,27)=1.636, p=.204, \eta_{\mathrm{p}}{ }^{2}=.057$, were not significant. For the low familiarity group, the main effect of Familiarity, $F(1$, $27)=0.918, p=.346, \eta_{\mathrm{p}}{ }^{2}=.033$, and interaction between factors, $F(1,27)=0.313, p=$ $.732, \eta_{\mathrm{p}}^{2}=.011$, were not significant.

Experiment 3. Median familiarity with 'familiar' celebrities was $68.8 \%$. Average familiarity for the high familiarity group was $81.4 \%(n=25, S D=8.1$, $\min =$ $70 \%, \max =100 \%$ ) and average familiarity for the low familiarity group was $53.2 \%(n=26, S D=9.7, \min =35 \%, \max =67.5 \%)$.

For the high familiarity group, both the main effect of Familiarity, $F(1,24)=2.232, p=$ $.148, \eta_{\mathrm{p}}^{2}=.085$, and interaction between factors, $F(1,24)=0.589, p=.624, \eta_{\mathrm{p}}{ }^{2}=.024$, were not significant. For the low familiarity group, the main effect of Familiarity, $F(1$, $25)=0.417, p=.524, \eta_{\mathrm{p}}{ }^{2}=.016$, and interaction between factors, $F(1,25)=0.625, p=$ $.601, \eta_{\mathrm{p}}^{2}=.024$, were not significant.

Experiment 4. Median familiarity with 'familiar' celebrities was 67.5\%. Average familiarity for the high familiarity group was $82 \%(n=26, S D=7.8, \min =70 \%, \max$ $=95 \%)$ and average familiarity for the low familiarity group was 50.8\% $(n=30, S D=$ $10.8, \min =27.5 \%, \max =67.5 \%)$.

For the high familiarity group, both the main effect of Familiarity, $F(1,25)=0.003, p=$ $.956, \eta_{\mathrm{p}}{ }^{2}=.000$, and interaction between factors, $F(1,25)=0.833, p=.370, \eta_{\mathrm{p}}{ }^{2}=.032$, 
were not significant. For the low familiarity group, the main effect of Familiarity, $F(1$, $29)=0.836, p=.368, \eta_{\mathrm{p}}{ }^{2}=.028$, and interaction between factors, $F(1,29)=2.594, p=$ $.118, \eta_{\mathrm{p}}^{2}=.082$, were not significant.

Experiment 5. Unlike the previous experiments, familiarity with the image set used in this experiment was much higher, with a median familiarity of $100 \%$. Consequently, the median split analysis instead compared the results of participants with $100 \%$ familiarity $(n=27)$ against participants with lower familiarity $(n=24)$. Average familiarity for the low familiarity group was $93.8 \%$ $(S D=4.3, \min =81.3 \%, \max =96.9 \%)$.

For the high familiarity group, the main effect of Familiarity was significant, $F(1,26)=$ $68.977, p<.001, \eta_{\mathrm{p}}{ }^{2}=.726$, with higher sensitivity for familiar faces than unfamiliar faces. However, there was also a significant interaction between Familiarity and Task Type, $F(1,26)=82.364, p<.001, \eta_{\mathrm{p}}^{2}=.760$. Follow up comparisons show significantly higher sensitivity for familiar faces than unfamiliar faces on the identity task, $t(26)=10.84, p<.001$, Cohen's $d=4.254$, but no significant differences between the familiar and unfamiliar faces on the image task, $t(26)=1.195, p=.178$, Cohen's $d=$ 0.468. The two-way interaction between Familiarity and Instruction Timing, $F(1,26)=$ $0.199, p=.659, \eta_{\mathrm{p}}{ }^{2}=.008$, and the three way interaction, $F(1,26)=1.061, p=.313, \eta_{\mathrm{p}}{ }^{2}$ $=.039$, were both not significant.

For the low familiarity group, we found the same pattern of results. There was a significant main effect of Familiarity, $F(1,23)=36.883, p<.001, \eta_{\mathrm{p}}{ }^{2}=.616$, with higher sensitivity for familiar than unfamiliar faces. Moreover, the interaction between Familiarity and Task Type was also significant, $F(1,23)=26.177, p<.001, \eta_{\mathrm{p}}{ }^{2}=.532$. Follow up comparisons again show significantly higher sensitivity for familiar faces than unfamiliar faces on the identity task, $t(23)=7.592, p<.001$, Cohen's $d=3.168$, but no significant differences between the familiar and unfamiliar faces on the image task, $t(23)<1$. The two-way interaction between Familiarity and Instruction Timing, $F(1,23)=0.025, p=.877, \eta_{\mathrm{p}}^{2}=.001$, and the three way interaction, $F(1,23)=0.003, p$ $=.954, \eta_{\mathrm{p}}^{2}=.000$, were both not significant. 


\section{Supplementary analysis 2: Response latency analysis}

The dependent variables in the main paper were sensitivity (d-prime) and response bias (criterion). This choice was motivated primarily by our research question: we were interested in the extent to which perceptual representations in short term memory contained image-specific details. The perceptual sensitivity was taken as a measure of the extent to which image-specific details has been stored in memory. Secondly, we chose this analysis to be consistent with the critical analysis in Armann et al. (2016), which our experiments were designed to follow up. The one exception to this was the visual search task reported in Experiment 4 where response latency is an important consideration (as explained in the main paper, targets were always present in these arrays and so accuracy had to be interpreted together with response latency).

In response to reviewers' comments, we also conducted a post-hoc analysis of participant's response latencies in Experiments 1,2, 3, and 5 and report the results below. This analysis was conducted to rule out speed-accuracy trade-offs as an account of the lack of differences between unfamiliar and familiar faces in image memory tasks.

Full details of this analysis are reported below. For each experiment, log transformations were applied to the median response latency for participants' correct trials in each condition, and then analysed using the same repeated measures ANOVA's used to analyse sensitivity and criterion in the main manuscript. For brevity, only the critical comparisons of this analysis that involve familiarity are reported.

To summarise, across 5 experiments we found one significant difference between unfamiliar and familiar faces in response latency to image memory tasks. This difference was found in Experiment 1, where participants took significantly longer to decide whether duplicated of familiar faces were present compared with unfamiliar faces. Consequently, we cannot rule out that the findings this experiment could be explained by a speed-accuracy trade-off. However, in Experiments 2 to 5 (note that Experiment 4 analysis reported in manuscript) we found no differences in response time between unfamiliar and familiar faces. Overall, this analysis provides very little support for the possibility that null effects of familiarity on sensitivity can be attributed to a speed accuracy trade-off. 
Experiment 1. There was a significant main effect of Familiarity, $F(1,55)=7.980, p=$ $.007, \eta_{\mathrm{p}}{ }^{2}=.127$, with participants taking longer on average with familiar faces than unfamiliar faces. The interaction between factors was not significant, $F(2,110)=1.203$, $p=.304, \eta_{\mathrm{p}}^{2}=.021$.

Experiment 2. The main effect of Familiarity, $F(1,55)=1.555, p=.218, \eta_{\mathrm{p}}{ }^{2}=$ .027 , and interaction between factors, $F(2,110)=2.177, p=.118, \eta_{\mathrm{p}}{ }^{2}=.038$, were both not significant.

Experiment 3. The main effect of Familiarity, $F(1,50)=0.010, p=.921, \eta_{\mathrm{p}}{ }^{2}=$ .000 , and interaction between factors, $F(3,150)=1.413, p=.241, \eta_{\mathrm{p}}{ }^{2}=.027$, were both not significant.

Experiment 5. There was a significant main effect of Familiarity, $F(1,50)=$ $9.631, p=.003, \eta_{\mathrm{p}}^{2}=.162$, with follow up comparisons showing slower responses for unfamiliar faces than familiar faces.

Critically, this effect was qualified by a significant interaction between Familiarity and Trial Type, $F(1,50)=22.611, p<.001, \eta_{\mathrm{p}}{ }^{2}=.311$. Follow up comparisons show significantly slower responses for unfamiliar faces than familiar faces on the identity task, $t(50)=4.846, p<.001$, Cohen's $d=1.372$, but no significant differences in latency between familiar faces and unfamiliar faces on the image task, $t(50)=0.737, p$ $=.465$, Cohen's $d=0.211$. The interaction between Familiarity and Instruction Timing, $F(1,50)=1.403, p=.242, \eta_{\mathrm{p}}{ }^{2}=.027$, and three-way interaction between all factors, $F(1,50)=0.081, p=.777, \eta_{\mathrm{p}}^{2}=.002$, were not significant. 